EPiC Series in Engineering
Volume 3, 2018, Pages 2375-2382
HIC 2018. 13th International
Conference on Hydroinformatics

\title{
Simulation of floating debris in violent shallow flows
}

\author{
Yan XIONG ${ }^{1}$, Samantha MAHAFFEY ${ }^{2}$, Qiuhua LIANG ${ }^{1,2^{*}}$ \\ ${ }^{1}$ State Key Laboratory of Hydrology-Water Resources and Hydraulic Engineering, Hohai \\ University, Nanjing, 210098, China \\ ${ }^{2}$ School of Engineering, Newcastle University, Newcastle upon Tyne, England, UK \\ xiongyan@hhu.edu.cn
}

\begin{abstract}
Storm surge and tsunami may induce violent shallow flows and carry dense debris, causing tremendous damage to human lives, buildings and structures. This work presents a series of laboratory experiments to investigate the debris movement in the extreme flows. Subsequently, a new modeling tool featured with a finite volume shock-capturing hydrodynamic model fully coupled with a discrete element model is introduced. A new coupling method totally depending on the hydrodynamic characteristics is proposed to simulate the complex debris-enriched floods induced by tsunamis or storm surges. The experimental measurements are used to validate the reliability of the coupled model. The numerical results agree satisfactorily with the experimental measurements, demonstrating the model's capability in simulating the complex fluid-debris interactions induced by violent shallow flows.
\end{abstract}

\section{Introduction}

In recent years, extreme marine disasters have become more frequent, e.g. the 2004 tsunami, 2011 Japan tsunami, 2012 hurricane Sandy, among many others, leading to catastrophic consequences to both people and their properties [1,2]. One of the striking features of these large-scale disaster events is the dense floating debris of different shapes and sizes carried forward overland by the advancing flood front. Field investigations imply that water flow alone is much less destructive than the debris-enriched flow during a tsunami run-up event [3].

However, the role of floating objects is rarely considered in the design codes for flooded and coastal areas. A number of physical modelling studies and theoretical analysis have been carried out to understand the basic behaviours of floating objects driven by surges. Riggs et al. [4] conducted fullscale and flume experiments to test the velocities and impact forces of a wood log and a shipping container under different flow conditions. Nistor et al. [5] and Goseberg et al. [6] introduced a series of basin experiments using a novel technique to track debris in real time. A horizontal platform with a vertical quay wall was constructed in the basin to reproduce the flow environment in a typical containerstacking harbour under tsunami attacks. The particle-tracking technique was enabled by the so-called 'Smart Debris' system that used motion sensors to record the trajectory, orientation and acceleration of

*xiongyan@hhu.edu.cn 
individual object. Initiated by a solitary wave, the maximum longitudinal displacements and spreading angles were recorded to study the detailed movement of down-scale shipping containers during a tsunami impact. Stolle et al. [7] performed flume experiments to examine the trajectory and velocity of the debris in dam-break hydrodynamic conditions. Their work showed that statistical characteristics of the debris motion could be determined and the lateral displacement of the debris could be evaluated using a normal distribution. However, these physical model experiments are limited to the laboratory environments and the understanding of the complex dynamics of floating debris is still at the preliminary discussion stage.

Theoretical analysis has also been carried out following laboratory experiments to better understand and describe the interaction between fluid flow and floating debris. Matsutomi et al. [8] carried out experiments to discuss the debris concentration at the surge front of a dam-break flow, and introduced a simple theoretical model. The model adopted the notion of the conventional bore theory to estimate the velocity of floating debris. The resistance induced by the floating bodies piling up at the surge front was found to increase bore depth but decrease debris velocity and bore front propagation. The debris velocity was found to be always less than or equal to the bore front velocity. Imamura et al. [9] conducted experiments in an open channel with cubic and rectangular blocks released in dam-break flows to investigate the boulder transport process during a tsunami. They proposed a theoretical model based on force balance of the boulder in contact with the ground, taking into account various transport modes, including saltation and rolling. Shafiei et al. [10] recorded the accelerations of debris in dambreak flow conditions utilizing an embedded tracking sensor. The equation of debris velocity was derived using a force balance theory assuming that debris entrainment begins after the leading edge of the bore passes the debris and that average stream-wise velocity behind the bore is constant. These theoretical analysis works have tried to understand and interpret the physical processes induced by the interaction between fluids and solids. But these studies are all developed under idealised or simplified conditions and are difficult to extend to wider practical engineering applications.

Numerical modelling as a more adaptable research and practical tool has been increasingly adopted to simulate the interaction between fluid and debris. In the field of river hydraulics, Hopkins and coworkers have attempted to develop strategies to couple shallow water models with discrete element models (DEM) to simulate the movement and accumulation of floating ice floes in channels [11]. However, the floating objects as observed during a tsunami or storm surge event are much more complex than ice floes, including objects of different size and shape, e.g. cars, houses, shipping containers, lamp poles, etc. Stockstill et al. [12] developed a one-way fluid-to-solid coupling model using a finite-element shallow water dynamic model and DEM to simulate the interaction between floating objects and navigational structures in the river channel. However, these models, lack of shockcapturing capability, cannot be directly applied to simulate the floating debris driven by the more violent flows caused by tsunami or storm surge.

Recently, attempts have also been made to couple Smoothed Particle Hydrodynamics (SPH) with DEM to simulate the dynamics of floating debris. For example, Ren et al. [13] presented a vertically 2D two-way SPH-DEM model to simulate the interaction between waves and the massive irregular blocks on a slope. The blocks were impacted by wave-induced hydrodynamic pressures, and the fluidsolid interaction was considered through an interactive force balance approach. Canelas et al. [14] introduced a fluid-solid interaction model by incorporating a distributed-contact DEM in the SPH framework. The boundary particles of the rigid bodies were taken as fluid particles for coupling and the contact forces were considered as a combination of a repulsion force and a damping force. The model was able to simulate the motion of individual particles and was validated comparing with experimental data [15]. Robb et al. [16] developed a two-way coupled SPH-DEM model for simulation of smallscale river ice jams and other similar problems involving free surface flows containing solids. Both fluid-solid and solid-solid interactions were resolved using a partial Riemann solver, and the force exerted by the solid on the fluid was calculated through SPH interpolation of neighbouring fluid particles. But, the numerical instability inherent to the SPH method was considered to be an issue that 
may lead to the fluctuation of calculated wave forces [13]. There are also several other attempts using SPH to simulate free-surface flows and the transport of moving bodies simultaneously $[17,18]$. However, these models also cannot be directly applied to predict the dynamics of floating debris induced by a tsunami or extreme flood event because SPH models are computationally too demanding for large-scale applications [19].

Therefore, there is a lack of the physically based understanding of precisely how floating objects interact with a propagating wave front; the development of sophisticated numerical models to represent the complex fluid-debris-structure interactions is still at a preliminary stage [19]. It is necessary to develop a computationally more efficient model suitable for robust simulation of the complex fluiddebris-structure interactions in a more violent hydrodynamic context. Herein, the aim of this work is to investigate the physical mechanism of the interaction between extreme flows and debris through laboratory experiments. The experimental data are then used to calibrate and validate a new fully coupled model consisting of a shock-capturing shallow flow model with a DEM, which has a great potential for practical engineering applications.

\section{Laboratory experiments}

(a)
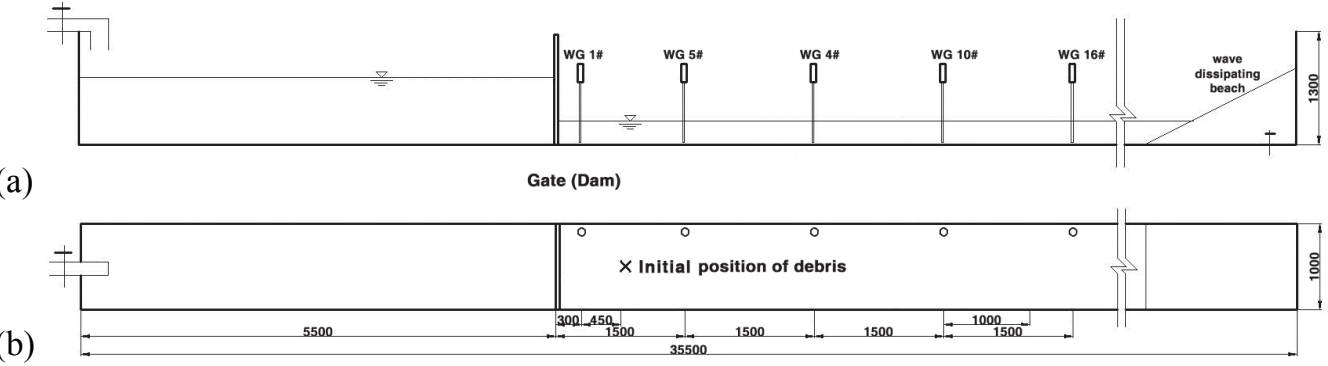

Figure 1: The flume and laboratory setup: (a) vertical view; (b) horizontal view (unit: mm).

(a)

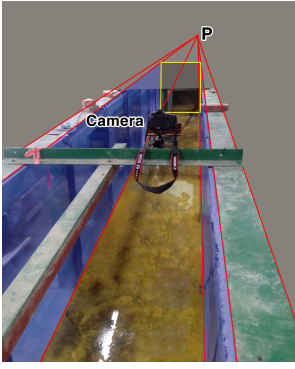

(b)

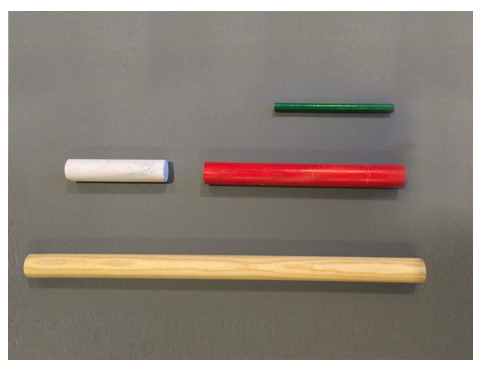

Figure 2: Photo of: (a) Camera and flume in one-point perspective; (b) Four cylindrical debris model.

A series of flume experiments were designed and carried out at the hydraulic laboratory in Hohai University, China. The flume is $35.5 \mathrm{~m}$ long, $1 \mathrm{~m}$ wide and $1.3 \mathrm{~m}$ deep. The bottom of the flume was painted in yellow. The two sidewalls of the flume are made of glasses supported and connected by green steel brackets, and both sidewalls are marked with tapes every $0.5 \mathrm{~m}$ during the experiments to better extract the data of debris positions. The experimental layout is illustrated in Figure 1 with a gate installed at the upstream section to separate the flume into two parts with different water levels. During the experiments, the extreme flow conditions were created by instantly opening (less than $0.15 \mathrm{~s}$ ) the gate to generate dam-break waves [20]. The initial position of debris model is set at $0.75 \mathrm{~m}$ downstream side of the gate ( $6.25 \mathrm{~m}$ from the left boundary). Five calibrated wave gauges (WG) installed along one side of the channel were used to record time histories of water depth without blocking the debris. 
A digital camera mounted at the top of the flume (1.5 m behind the WG $16 \#$ ) was used to capture the flow dynamics and the motions of debris, as shown in Figure 2(a). The size of the record videos was set to be 1028 pixel $\times 720$ pixel with 50 frames per second. Figure 2(a) also shows the composition principle of one-point perspective used in following graphic analysis. The part of the photo except the flume is covered in grey for clearer performance, and all auxiliary lines were made in the graphics processing software (Photoshop) to make sure straight. The flume is made up of lines either directly parallel with the camera's sight (red lines) or directly perpendicular (horizontal yellow lines), and all parallel red lines converge at a vanishing point $(\mathrm{P})$. Hence, the photo of flume conforms to one-point perspective principle and the video token during the experiments can be then analysed in this way. The translucent blue covering area represents the glass sidewalls of the flume, and the rectangular shadow plane in the yellow frame is the plane of the gate. Figure 2(b) shows four different sizes of cylindrical woody debris used in the physical experiments. Their colours and sizes are listed in Table 1. Over 15 experimental tests were performed with different debris and different water depth upstream and downstream of the gate, including the dry bed cases. Debris of different sizes was used in each test. The initial water levels upstream and downstream of the gate of all tests and corresponding debris models are shown in Table 2.

\begin{tabular}{|c|c|c|c|}
\hline Debris & Colour & Length (m) & Radius (m) \\
\hline D1 & Green & 0.12 & 0.01 \\
\hline D2 & White & 0.10 & 0.02 \\
\hline D3 & Red & 0.20 & 0.02 \\
\hline D4 & Nude & 0.4 & 0.02 \\
\hline Table 1: & ailed information of ex & erimental debris & \\
\hline $\begin{array}{c}\text { Test } \\
\text { Number }\end{array}$ & $\begin{array}{l}\text { Initial upstream } \\
\text { water level (m) }\end{array}$ & $\begin{array}{l}\text { Initial downstream } \\
\text { water level }(m)\end{array}$ & Debris \\
\hline Case 1 & \multirow{2}{*}{0.2} & 0 & D2, D3, D4 \\
\hline Case 2 & & 0.1 & $\mathrm{D} 1, \mathrm{D} 2, \mathrm{D} 3, \mathrm{D} 4$ \\
\hline Case 3 & \multirow{2}{*}{0.3} & 0 & D2, D3, D4 \\
\hline Case 4 & & 0.1 & $\mathrm{D} 1, \mathrm{D} 2, \mathrm{D} 3, \mathrm{D} 4$ \\
\hline Case 5 & \multirow{2}{*}{0.4} & 0 & $\mathrm{D} 2, \mathrm{D} 3$ \\
\hline Case 6 & & 0.1 & $\mathrm{D} 2, \mathrm{D} 3$ \\
\hline
\end{tabular}

Table 2: Initial water levels and corresponding debris models

\section{Coupled shallow water and DEM model}

In this section, the fully coupled model consisting of a shallow water equation model and a DEM is introduced for simulation of debris-enriched shallow flows.

\subsection{Shock-capturing shallow water equation model}

The 2D non-linear shallow water equations (SWEs) have been widely adopted to describe the hydrodynamics of shallow water flows including tsunamis and storm surges. The dimensionality of the 2D SWEs can be reduced further to $1 \mathrm{D}$ if the flow is homogeneous in one of the horizontal dimensions: $\frac{\partial \mathbf{q}}{\partial t}+\frac{\partial \mathbf{f}}{\partial x}=\mathbf{s}$

where $t$ and $x$ represent respectively the time and $x$ coordinates (flow direction), and $\mathbf{q}, \mathbf{f}$ and $\mathbf{s}$ are the vectors containing the flow variables, fluxes and source terms. The vectors are provided as follows

$\mathbf{q}=\left[\begin{array}{ll}\eta & u h\end{array}\right]^{\mathrm{T}} ; \mathbf{f}=\left[\begin{array}{ll}u h & u^{2} h+g\left(\eta^{2}-2 \eta z_{b}\right) / 2\end{array}\right]^{\mathrm{T}} ; \mathbf{s}=\left[\begin{array}{ll}0 & -\tau_{b x} / \rho \\ -g \eta \partial z_{b} / \partial x+S_{v x}-S_{p x}\end{array}\right]^{\mathrm{T}}$ 
where $\eta$ and $z_{b}$ represent the water surface elevation and bed elevation above datum with $h=\eta-z_{b}$ being the total water depth; $u$ is the depth-averaged velocity; $g$ is the acceleration due to gravity; $\rho$ denotes the water density; $-\partial z_{b} / \partial x$ defines the bed slope; $\tau_{b x}$ is the bed friction stress; $S_{v x}$ is the turbulent (viscous) term; and $S_{p x}$ contains the extra forces to facilitate the balanced force coupling with a DEM model, which will be introduced in Section 3.3.

The above SWEs are solved using a second-order finite volume Godunov-type scheme implemented with an HLL approximate Riemann solver for evaluating interface fluxes. The second-order accuracy in both space and time is achieved through a two-step MUSCL-Hancock method. Detailed implementation of the numerical method can be found in [21].

\subsection{Discrete element model}

In a discrete element model [22], the following discretized equations are normally used to calculate the translational and rotational motions of the elements:

$m_{i} \frac{d \mathbf{w}_{i}}{d t}=\mathbf{F}_{i}^{p}+\mathbf{F}_{i}^{f}+\mathbf{F}_{i}^{g} ; \quad I_{i} \frac{d \mathbf{\omega}_{i}}{d t}=\mathbf{T}_{i}^{p}+\mathbf{T}_{i}^{f}$

where $i$ is the index of element/particle, $m_{i}$ and $I_{i}$ are the mass and moment of element $i ; \mathbf{w}_{i}$ and $\boldsymbol{\omega}_{i}$ are the velocity and angular velocity; $\mathbf{F}_{i}^{p}$ and $\mathbf{T}_{i}^{p}$ are the sum of contact forces and torques acting on element $i$ by other elements or objects; $\mathbf{F}_{i}^{f}$ and $\mathbf{T}_{i}^{f}$ are the sum of fluid forces and torques on the element; and $\mathbf{F}_{i}^{g}$ represents the gravity force.

For each particle, the combined forces and torques are used to calculate the acceleration, velocity and displacement at every time step. In this preliminary study, circles or circular discs are adopted to idealize the floating objects.

\subsection{Coupled model}

Buoyancy, hydrostatic and hydrodynamic forces are taken into account when coupling the SWE model with DEM. In the vertical direction, the buoyancy is calculated as

$F_{i}^{b}=\rho g V_{i}$

where $V_{i}$ is the volume of fluid displaced by the particle under consideration, which may be quantified differently for particles that are "completely submerged" and "floated".

In the horizontal direction, hydrostatic and hydrodynamic forces are calculated from the flow variables predicted by the SWE model. The total pressure $p_{i}^{h}$ at an arbitrary point on particle $i$ is given by

$p_{i}^{h}=p_{i}^{s}+p_{i}^{d}=\rho g z+\rho\left(\beta u-w_{i}\right)^{2}$

where $p_{i}^{s}$ and $p_{i}^{d}$ are the static and dynamic components of the point pressure, respectively; $z$ is the water depth from the surface to the point of interest; $u$ and $w_{i}$ are the depth-averaged flow velocity and the velocity of particle $i$, obtained respectively from the SWE model and DEM; $\beta$ is a correction factor introduced to better estimate the surface flow velocity. The total fluid impact moving debris/particle $i$ can be obtained as follows

$$
F_{i}^{h}=p_{i B}^{h} S_{B}-p_{i F}^{h} S_{F}
$$

where the subscripts, $B$ and $F$, indicate the 'Back' and 'Front' of the particle, and $S_{B}$ and $S_{F}$ are the areas of 'Back' side and 'Front' side of the particle submerged in the water. The SWE model will be coupled to the DEM by adding the counter-forces as source terms in the governing SWEs as given in Eq. (2), i.e. 
$S_{p x}=p_{i}^{h} / \rho$

\section{Results and discussion}

This section presents and discusses the laboratory observations, which are then used to validate the coupled floating debris model introduced in the last section.

\subsection{Laboratory Observations}

Videos have been recorded during the experiments to capture the flow and debris process. Each frame of the video files was extracted for one-point perspective analyse. To reflect the tape marks on the side wall, the vertical yellow solid lines on the side wall were drawn digitally to indicate the distances from the gate. The position of the horizontal red solid lines were determined by the intersections of the yellow lines the boundaries of the bottom of the flume. In order to determine the displacement of the floating object moving in the flume, the relationship between the two red lines was also analysed by perspective principle. Figure 3 presents the screenshots of some representative tests at different times after the gate was opened. The wooden object is outlined in white circle to highlight its location. The blue dotted line represents the starting position of the floating object. In order to show more clearly, only the red solid lines with $1 \mathrm{~m}$ interval from the gate are drawn, which means from the top of each screenshot, the red solid line respectively represents $x=5.5 \mathrm{~m}, 6.5 \mathrm{~m}, 7.5 \mathrm{~m}, 8.5 \mathrm{~m}$, and so on.

(a)
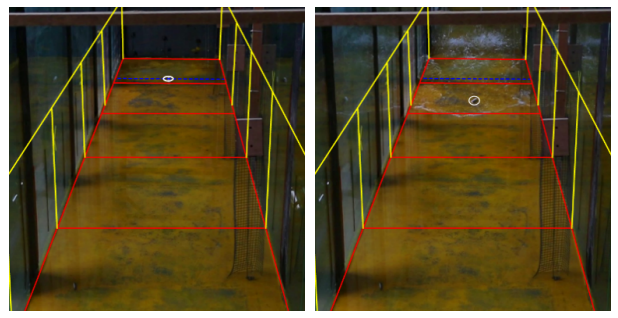

(b)
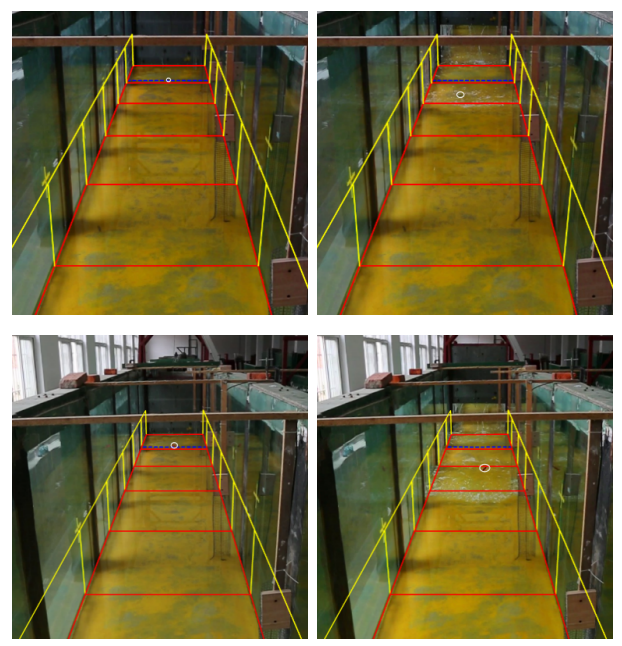
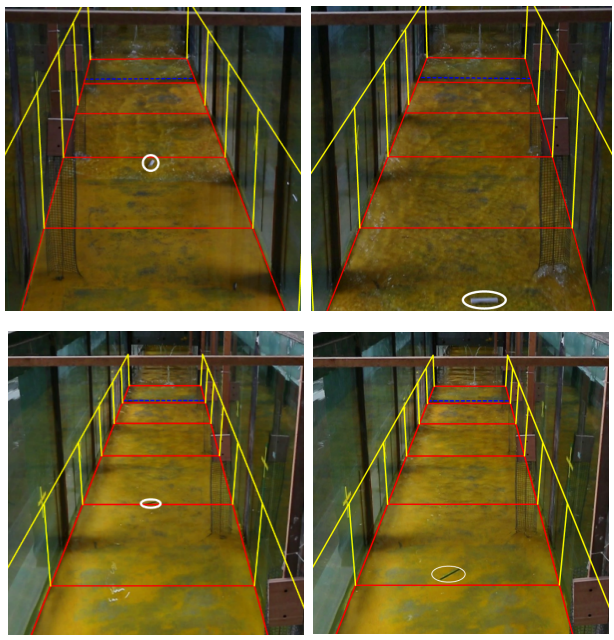

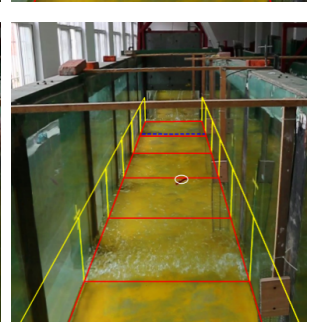

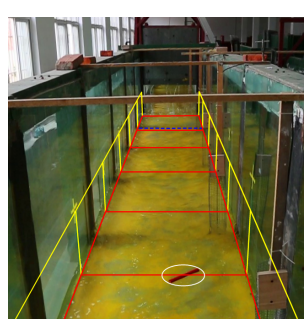


(d)
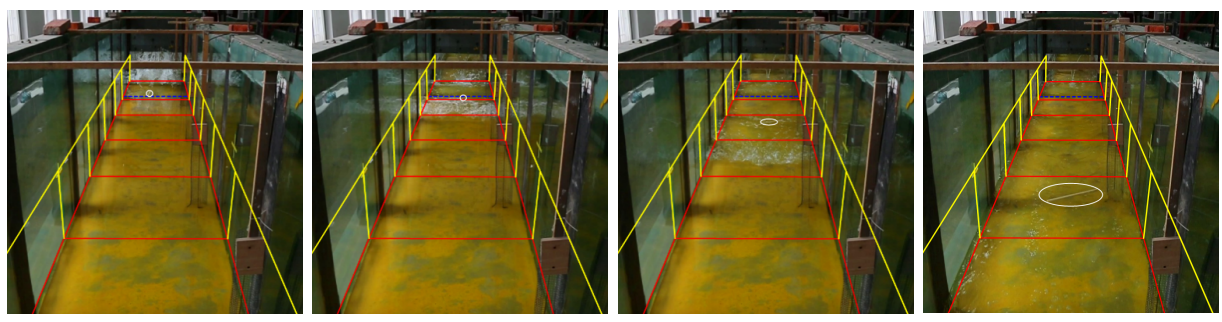

(e)
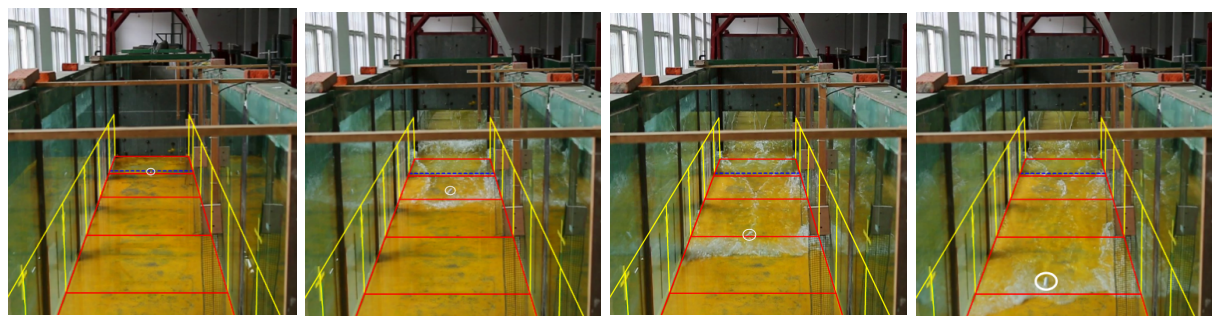

Figure 3: Screenshots of the video recording the motion of debris at different time: (a) Case 1 with D2 at $t=0 \mathrm{~s}, 1 \mathrm{~s}, 2 \mathrm{~s}$ and 3s; (b) Case 2 with D1 at $t=0 \mathrm{~s}, 2 \mathrm{~s}, 6 \mathrm{~s}$ and $8 \mathrm{~s}$; (c) Case 4 with D3 at $t=0 \mathrm{~s}, 2 \mathrm{~s}, 3 \mathrm{~s}$ and $5 \mathrm{~s}$; (d) Case 4 with D4 at $t=0 \mathrm{~s}, 1 \mathrm{~s}, 2 \mathrm{~s}$ and $4 \mathrm{~s}$; (e) Case 5 with D2 at $t=0 \mathrm{~s}$, $0.8 \mathrm{~s}, 1.3 \mathrm{~s}$ and $1.6 \mathrm{~s}$.

\subsection{Numerical Model Validation}

The new coupled model has been successfully applied to reproduce the aforementioned flume experiments. For example, Figures 4(a)\&(b) present the results of the simulation carried out for a drybed test and a wet-bed test, respectively. The time serious of water depth at WG 5\# and the positions of the floating object in the $x$-direction are compared satisfactorily with experimental measurements. Successful reproduction of these experimental tests demonstrates the model's ability in simulating the complex motions of floating debris driven by violent dam-break flows.

(a)
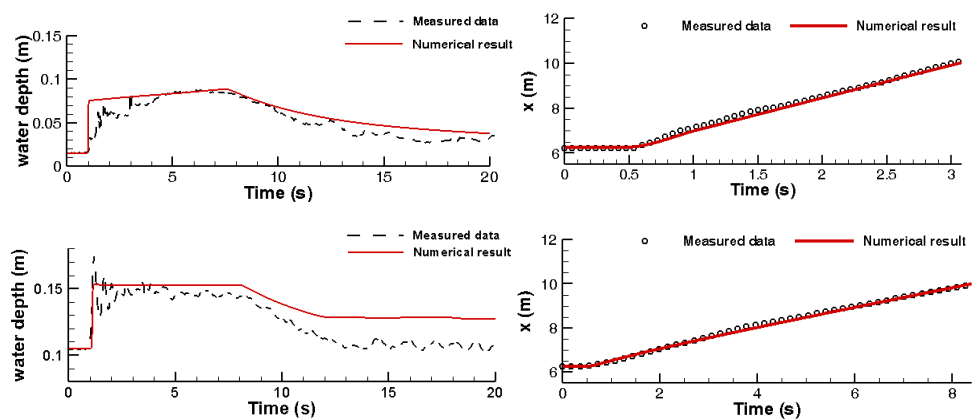

Figure 4: Predicted and measured time serious of water depth and $x$-position of the floating object for (a) Case 1 with D2; (b) Case 2 with D3.

\section{Conclusion}

In this paper, physical experiments have been conducted to observe and better understand the physical processes of floating debris moving in extreme hydrodynamic conditions. The trajectory data of debris is extracted by analysing video using graphic composition principle. A new modelling strategy is proposed to simulate the complex behaviours of floating debris driven by shallow flows under extreme hydrodynamic conditions. A shock-capturing finite volume Godunov-type numerical model and a DEM are coupled through the balance of buoyancy force, hydrostatic and hydrodynamic force. 
The new coupled model has been successfully validated against flume experimental tests, showing the model's ability to quantitatively capture the dynamics of the floating objects. The coupled model has great potential for practical applications.

\section{References}

A. Hatzikyriakou, N. Lin, J. Gong, S. Xian, X. Hu, and A. Kennedy, "Component-based vulnerability analysis for residential structures subjected to storm surge impact from Hurricane Sandy," Natural Hazards Review, vol. 17, p. $05015005,2015$.

F. Zhou, H. Cui, and S. ABE, "Inspection report of the disaster of the East Japan earthquake by Sino-Japanese joint mission," Building Sturcture, vol. 42, pp. 1-20, 2012.

H. H.-j. Yeh, I. Robertson, and J. Preuss, Development of design guidelines for structures that serve as tsunami vertical evacuation sites vol. 4: Washington State Department of Natural Resources, Division of Geology and Earth Resources, 2005.

H. Riggs, D. Cox, C. Naito, M. Kobayashi, P. P. Aghl, H.-S. Ko, et al., "Water-driven debris impact forces on structures: Experimental and theoretical program," in ASME 2013 32nd International Conference on Ocean, Offshore and Arctic Engineering, 2013, pp. V001T01A059-V001T01A059.

I. Nistor, N. Goseberg, J. Stolle, T. Mikami, T. Shibayama, R. Nakamura, et al., "Experimental Investigations of Debris Dynamics over a Horizontal Plane," Journal of Waterway, Port, Coastal, and Ocean Engineering, vol. 143, p. 04016022, 2016.

N. Goseberg, I. Nistor, T. Mikami, T. Shibayama, and J. Stolle, "Nonintrusive spatiotemporal smart debris tracking in turbulent flows with application to debris-laden tsunami inundation," Journal of Hydraulic Engineering, vol. 142, p. 04016058, 2016.

J. Stolle, N. Goseberg, I. Nistor, and E. Petriu, "Probabilistic Investigation and Risk Assessment of Debris Transport in Extreme Hydrodynamic Conditions," Journal of Waterway, Port, Coastal, and Ocean Engineering, vol. 144, p. 04017039, 2017.

H. Matsutomi, M. Fujii, and T. Yamaguchi, "Experiments and development of a model on the inundated flow with floating bodies," in PROCEEDINGS OF COASTAL ENGINEERING, JSCE, 2007, pp. 226-230.

F. Imamura, K. Goto, and S. Ohkubo, "A numerical model for the transport of a boulder by tsunami," Journal of Geophysical Research: Oceans, vol. 113, 2008.

S. Shafiei, B. W. Melville, A. Y. Shamseldin, S. Beskhyroun, and K. N. Adams, "Measurements of tsunami-borne debris impact on structures using an embedded accelerometer," Journal of Hydraulic Research, vol. 54, pp. 435-449, 2016.

M. A. Hopkins and S. F. Daly, "Recent advances in discrete element modeling of river ice," in Proceedings of the 12th Workshop on the Hydraulics of Ice Covered Rivers, Edmonton, Alta, 2003, pp. 19-20.

R. L. Stockstill, S. F. Daly, and M. A. Hopkins, "Modeling floating objects at river structures," Journal of Hydraulic Engineering, vol. 135, pp. 403-414, 2009.

B. Ren, Z. Jin, R. Gao, Y.-x. Wang, and Z.-1. Xu, "SPH-DEM modeling of the hydraulic stability of 2D blocks on a slope," Journal of Waterway, Port, Coastal, and Ocean Engineering, vol. 140, p. 04014022, 2013.

R. Canelas, R. M. Ferreira, A. J. Crespo, and J. Domínguez, "A generalized SPH-DEM discretization for the modelling of complex multiphasic free surface flows," in Proceedings of the 8th International SPHERIC Workshop, 2013, pp. 7479.

R. Canelas, A. A. J. C. Crespo, J. M. Domínguez, M. Gómez-Gesteira, and M. L. F. Rui, "Resolved Simulation of a Granular-Fluid Flow with a Coupled SPH-DCDEM Model," Journal of Hydraulic Engineering, vol. 143, 2017.

D. M. Robb, S. J. Gaskin, and J.-C. Marongiu, "SPH-DEM model for free-surface flows containing solids applied to river ice jams," Journal of Hydraulic Research, vol. 54, pp. 27-40, 2016.

R. B. Canelas, J. M. Domínguez, A. J. C. Crespo, M. Gómez - Gesteira, and R. M. L. Ferreira, "A Smooth Particle Hydrodynamics discretization for the modelling of free surface flows and rigid body dynamics," International Journal for Numerical Methods in Fluids, vol. 78, pp. 581-593, 2015.

A. Amicarelli, R. Albano, D. Mirauda, G. Agate, A. Sole, and R. Guandalini, "A Smoothed Particle Hydrodynamics model for 3D solid body transport in free surface flows," Computers \& fluids, vol. 116, pp. 205-228, 2015.

I. Nistor, N. Goseberg, and J. Stolle, "Tsunami-Driven Debris Motion and Loads: A Critical Review," Frontiers in Built Environment, vol. 3, p. 2, 2017.

Q. Liang, K.-c. Chen, H. Jingming, Y. Xiong, W. Gang, and J. Qiang, "Hydrodynamic modelling of flow impact on structures under extreme flow conditions," Journal of Hydrodynamics, Ser. B, vol. 28, pp. 267-274, 2016.

Q. Liang and A. G. Borthwick, "Adaptive quadtree simulation of shallow flows with wet-dry fronts over complex topography," Computers \& Fluids, vol. 38, pp. 221-234, 2009.

P. A. Cundall and O. D. Strack, "A discrete numerical model for granular assemblies," geotechnique, vol. 29, pp. 47-65, 1979. 